\title{
In vitro cytotoxicity of Manville Code 100 glass fibers: Effect of fiber length on human alveolar macrophages
} Patti C Zeidler-Erdely1, William J Calhoun², Bill T Ameredes², Melissa P Clark², Gregory J Deye ${ }^{3}$, Paul Baron ${ }^{3}$, William Jones ${ }^{4}$, Terri Blake ${ }^{1}$ and Vincent Castranova*1

Address: ${ }^{1}$ Health Effects Laboratory Division, National Institute for Occupational Safety and Health, Morgantown, WV, USA, ${ }^{2}$ AAARC, Division of Pulmonary, Allergy, and Critical Care Medicine, University of Pittsburgh, Pittsburgh, PA, USA, ${ }^{3}$ Division of Applied Research and Technology, National Institute for Occupational Safety and Health, Cincinnati, OH, USA and ${ }^{4}$ Division of Respiratory Disease Studies, National Institute for Occupational Safety and Health, Morgantown, WV, USA

Email: Patti C Zeidler-Erdely - paz9@cdc.gov; William J Calhoun - wjcalhou@utmb.edu; Bill T Ameredes - btamered@utmb.edu; Melissa P Clark - clarkmp@upmc.edu; Gregory J Deye - gjd2@cdc.gov; Paul Baron - pab2@cdc.gov; William Jones - vic1@cdc.gov; Terri Blake - trblake@earthlink.net; Vincent Castranova* - vic1@cdc.gov

* Corresponding author

Published: 28 March 2006

Particle and Fibre Toxicology2006, 3:5 doi:10.1 186/1743-8977-3-5
Received: 05 December 2005

Accepted: 28 March 2006

This article is available from: http://www.particleandfibretoxicology.com/content/3/I/5

(c) 2006Zeidler-Erdely et al; licensee BioMed Central Ltd.

This is an Open Access article distributed under the terms of the Creative Commons Attribution License (http://creativecommons.org/licenses/by/2.0), which permits unrestricted use, distribution, and reproduction in any medium, provided the original work is properly cited.

\begin{abstract}
Background: Synthetic vitreous fibers (SVFs) are inorganic noncrystalline materials widely used in residential and industrial settings for insulation, filtration, and reinforcement purposes. SVFs conventionally include three major categories: fibrous glass, rock/slag/stone (mineral) wool, and ceramic fibers. Previous in vitro studies from our laboratory demonstrated length-dependent cytotoxic effects of glass fibers on rat alveolar macrophages which were possibly associated with incomplete phagocytosis of fibers $\geq 17 \mu \mathrm{m}$ in length. The purpose of this study was to examine the influence of fiber length on primary human alveolar macrophages, which are larger in diameter than rat macrophages, using length-classified Manville Code 100 glass fibers $(8,10,16$, and $20 \mu \mathrm{m})$. It was hypothesized that complete engulfment of fibers by human alveolar macrophages could decrease fiber cytotoxicity; i.e. shorter fibers that can be completely engulfed might not be as cytotoxic as longer fibers. Human alveolar macrophages, obtained by segmental bronchoalveolar lavage of healthy, non-smoking volunteers, were treated with three different concentrations (determined by fiber number) of the sized fibers in vitro. Cytotoxicity was assessed by monitoring cytosolic lactate dehydrogenase release and loss of function as indicated by a decrease in zymosan-stimulated chemiluminescence.

Results: Microscopic analysis indicated that human alveolar macrophages completely engulfed glass fibers of the $20 \mu \mathrm{m}$ length. All fiber length fractions tested exhibited equal cytotoxicity on a per fiber basis, i.e. increasing lactate dehydrogenase and decreasing chemiluminescence in the same concentration-dependent fashion.

Conclusion: The data suggest that due to the larger diameter of human alveolar macrophages, compared to rat alveolar macrophages, complete phagocytosis of longer fibers can occur with the human cells. Neither incomplete phagocytosis nor length-dependent toxicity was observed in fiber-exposed human macrophage cultures. In contrast, rat macrophages exhibited both incomplete phagocytosis of long fibers and length-dependent toxicity. The results of the human and rat cell studies suggest that incomplete engulfment may enhance cytotoxicity of fiber glass. However, the possibility should not be ruled out that differences between human versus rat macrophages other than cell diameter could account for differences in fiber effects.
\end{abstract}




\section{Background}

Synthetic vitreous fibers (SVFs) are inorganic noncrystalline materials widely used in residential and industrial settings for insulation, filtration, and reinforcement purposes. SVFs conventionally include three major categories: fibrous glass, rock/slag/stone (mineral) wool, and ceramic fibers [1]. The chemical composition of fibrous materials is known to play a role in fiber-induced toxicity as fiber biodurability directly correlates with pathogenic potential in rodents [2], but it has also been suggested that fiber length is an important factor. In the past, the study of fiber length as a cause of toxicity has been complicated by the inability to obtain pure size-selected fiber samples. However, the development of the dielectrophoretic classifier by Baron and colleagues has aided in the study of monodisperse size-selected fiber samples on lung cell activation and toxicity [3]. This classifier separates fibers by length using dielectrophoresis that involves the movement of neutral particles in a gradient electric field $[3,4]$. Rodent macrophage toxicity and activation have previously been demonstrated in vitro in our laboratory using these length-classified fibers and indeed, fiber length was an important determinant [5-7].

Frustrated or incomplete phagocytosis has been implicated as a mechanism of fiber-induced cytotoxicity. This process involves repeated attempts by a phagocyte to engulf a fiber longer than its diameter, thereby possibly enhancing its respiratory burst activity [8]. In comparison to short fibers that are fully engulfed, longer fibers may cause sustained cellular activation and increase phagocyte recruitment into the airspace, subsequently increasing lung oxidant burden [9-11]. Indeed, several in vivo and in vitro rodent studies suggest longer fibers are more pathogenic than short fibers [12-14]. However, macrophage size is relevant when investigating fiber toxicity because human alveolar macrophages are larger in size than rat alveolar macrophages, approximately 18 and $13 \mu \mathrm{m}$ in average diameter, respectively [15]. Therefore, the purpose of this study was to examine the influence of fiber length on isolated primary human alveolar macrophages, which are larger in diameter than rat macrophages, using length-classified Manville Code 100 (JM-100) glass fibers $(8,10,16$, and $20 \mu \mathrm{m})$. Respiratory burst activity and leakage of cytosolic lactate dehydrogenase ( $\mathrm{LDH}$ ) were used as parameters of activation and toxicity, respectively. Microscopic analysis was also conducted to determine if frustrated phagocytosis had occurred. A comparison to results obtained using the rat alveolar macrophage is made. Since this investigation employed a static rather than a flow system, issues of fiber solubility were not addressed.

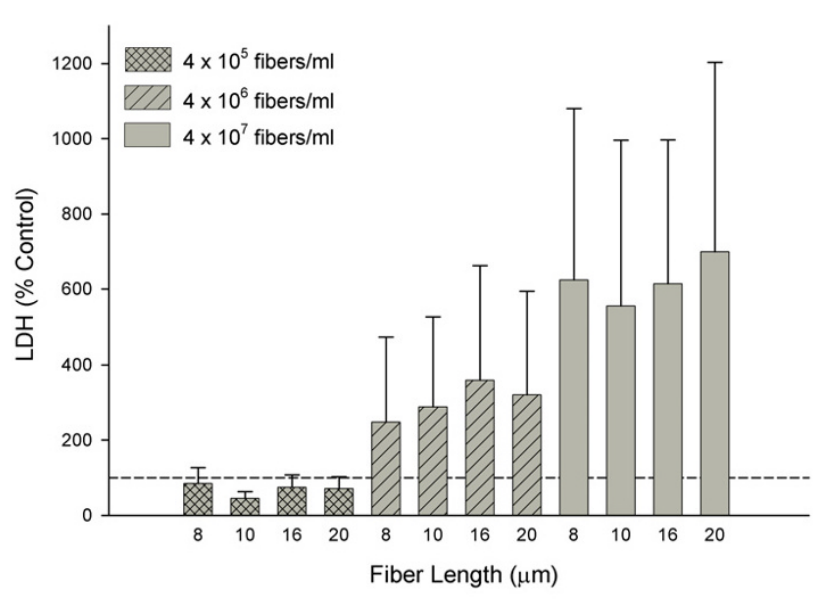

\section{Figure I}

Lactate dehydrogenase release from primary human alveolar macrophages $\left(I \times 10^{5}\right.$ cells/well) following exposure to JM100 glass fibers for 18 hours. Data are presented as percent control from $100 \%$. Bars represent mean values \pm S.E. of three independent experiments. No significant difference was found among fiber length groups of a given concentration.

\section{Results}

\section{Glass fiber induced LDH}

Figure 1 shows glass fiber-induced cytotoxicity on human alveolar macrophages as measured by the LDH assay 18 hours post-treatment in vitro. The fiber lengths tested (8, 10,16 , and $20 \mu \mathrm{m}$ ) all exhibited equal cytotoxic effects. These effects were not significantly different among fiber length groups of the same concentration, i.e., fiber length did not affect cytotoxicity over the range of lengths evaluated. A trend toward a concentration-response was observed although this did not achieve statistical significance. Table 1 depicts the effects of glass fiber length on rat alveolar macrophages as reported previously [7]. Significant toxicity, measured as \% of total LDH, occurred with the long $(17 \mu \mathrm{m})$ glass fiber, but a shorter fiber $(7 \mu \mathrm{m})$ showed little or no toxic effect.

\section{Glass fiber induced inhibition of zymosan-stimulated chemiluminescence}

Zymosan-stimulated chemiluminescence, reported as \% control, is depicted in Figure 2. Zymosan, a particulate $\beta$ glucan from yeast cell walls, was used to stimulate reactive species production in the human alveolar macrophages exposed to glass fibers for 18 hours in vitro. Data show a concentration-dependent decrease in macrophage activation (i.e. reactive species production) with increasing fiber concentration. Fiber length did not significantly affect human alveolar macrophage function. It is of interest to note the lowest concentration of fibers caused a priming ( $71 \%$ increase in activation) of the human cells to release reactive species in response to zymosan while the highest 
Table I: Relative Toxicity (\% of total LDH) of Rat Alveolar Macrophages Exposed to Length Classified Glass Fibers

\begin{tabular}{ccc}
\hline Fiber Length $(\mu \mathrm{m})$ & \multicolumn{2}{c}{ Log $($ fiber \#/ml) } \\
\cline { 2 - 3 } & $10^{6}(\mathrm{I}: 2.5$ fiber:cell) & $10^{7}$ (4: I fiber:cell) \\
\hline 3 & $\mathrm{ND}$ & $\mathrm{ND}$ \\
4 & $\mathrm{ND}$ & $\mathrm{ND}$ \\
7 & $\mathrm{ND}$ & $2 \%$ \\
17 & $19 \%^{*}$ & $45 \%^{*}$ \\
\hline
\end{tabular}

Note: Adapted from Blake et al., 1998; ND is not detected ; LDH is lactate dehydrogenase; * represents a significant difference from control

concentration caused an $84 \%$ loss of activation. Table 2, adapted from Blake et al., represents the percent decreased activation versus control observed in rat macrophages following glass fiber exposure for 18 hours [7]. In contrast to the human cells, the rat macrophages show distinctly decreased oxidative function with increasing fiber length, with the $17 \mu \mathrm{m}$ fiber exhibiting a stronger effect $(100 \%$ loss of activation) than the $7 \mu \mathrm{m}$ fiber $(15 \%$ reduction of activation). In addition, no cellular priming was observed in the rat macrophages as compared to the human.

\section{Microscopic examination of primary human alveolar macrophages}

Figure 3, panel A, demonstrates the ability of human macrophages to engulf $8 \mu \mathrm{m}$ glass fibers in vitro. Figure 3, panel B, demonstrates that human macrophages can successfully engulf $20 \mu \mathrm{m}$ glass fibers. Data from Blake et al. revealed incomplete phagocytosis by rat alveolar macrophages occurred with glass fibers $\geq 17 \mu \mathrm{m}$ in length [7]. These results suggest human alveolar macrophages can completely phagocytize fiber lengths that rat alveolar macrophages can not. Therefore, frustrated phagocytosis and its possible effects do not appear to be a factor with human alveolar macrophages exposed to fibers $\leq 20 \mu \mathrm{m}$ in length.

\section{Discussion}

The present study employed an in vitro system to expose primary human alveolar macrophages to monodisperse JM-100 glass fibers of different target lengths. Using this system, human alveolar macrophages were assessed for length-dependent cellular effects and results were compared to previous data obtained with rat alveolar macrophages. The data presented here reveal differences in the responses of rat versus human macrophages to glass fibers of various lengths.

Previous studies have shown that rat alveolar and mouse peritoneal macrophages attempt to engulf glass fibers and that successful phagocytosis is influenced by fiber length $[5,7]$. Short $(7 \mu \mathrm{m})$ glass fibers were completely engulfed,

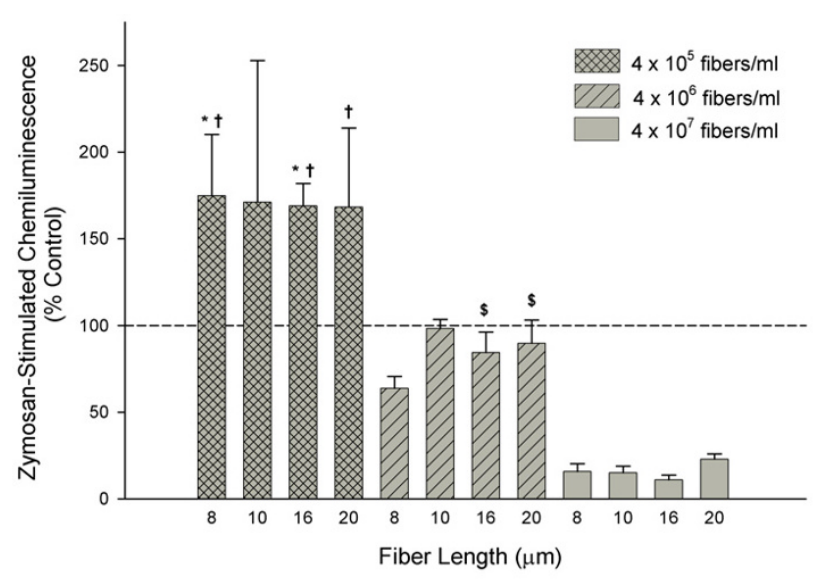

Figure 2

Inhibition of zymosan-stimulated primary human alveolar macrophage chemiluminescence following an 18 hour exposure to JM- 100 glass fibers. Data are presented as percent control from $100 \%$. Bars represent mean values \pm S.E of three independent experiments. $*$ - indicates a significant difference between the lowest $\left(4 \times 10^{5}\right.$ fibers $/ \mathrm{ml}$ or I:I fiber:cell) and intermediate ( $4 \times 10^{6} \mathrm{fibers} / \mathrm{ml}$ or $8:$ : fiber:cell) fiber concentrations, $f$ - between the lowest and highest $(4 \times$ $10^{7}$ fibers $/ \mathrm{ml}$ or 80 : I fiber:cell) fiber concentrations, and $\$$ between the intermediate and highest fiber concentrations of the same fiber length $(p \leq 0.05)$. Fiber length did not affect human alveolar macrophage function.

whereas long $(17 \mu \mathrm{m})$ glass fibers were only partially engulfed. Human alveolar macrophages have a larger diameter than the rat counterpart; therefore, it was hypothesized that these phagocytes would completely engulf longer fibers and the absence of frustrated phagocytosis would attenuate cytotoxicity. Overall, the data supported this hypothesis.

Human alveolar macrophage function, measured as zymosan-stimulated chemiluminescence, was significantly affected by fiber concentration but not fiber length over the range of $8-20 \mu \mathrm{m}$ (Figure 2). The lowest of three fiber concentrations $\left(4 \times 10^{5}\right.$ fibers $/ \mathrm{ml}$ or $1: 1$ fiber:cell ratio) primed the cellular response to zymosan while the highest concentration $\left(4 \times 10^{7}\right.$ fibers $/ \mathrm{ml}$ or $80: 1$ fiber:cell ratio) decreased this response from control. The activation level of cells exposed to the intermediate fiber concentration $\left(4 \times 10^{6}\right.$ fibers $/ \mathrm{ml}$ or $8: 1$ fiber:cell ratio) was similar to that of controls). In contrast, our previous findings showed no increased priming effect of JM-100 glass fibers in rat alveolar macrophages at any concentration. This differential response between human and rat cells may be the result of several possible factors. First, fiber to cell ratio has been shown in our laboratory to be important in the activation of rodent macrophages. Prior studies revealed a fiber to cell ratio of at least 5:1 is required for significant 
Table 2: Relative Toxicity (\% Decreased Activation vs. Control) of Rat Alveolar Macrophages Exposed to Length Classified Glass Fibers

\begin{tabular}{ccc}
\hline Fiber Length $(\mu \mathrm{m})$ & \multicolumn{2}{c}{ Log $($ fiber \#/ml) } \\
\cline { 2 - 3 } & $10^{6}(\mathrm{I}: 2.5$ fiber:cell) & $10^{7}$ (4:I fiber:cell) \\
\hline 3 & $\mathrm{ND}$ & $\mathrm{ND}$ \\
4 & $\mathrm{ND}$ & $\mathrm{ND}$ \\
7 & $\mathrm{ND}$ & $15 \%$ \\
17 & $53 \%^{*}$ & $100 \%^{*}$ \\
\hline
\end{tabular}

Note: Adapted from Blake et al., 1998; ND is not detected;*

represents a significant decrease from control

tumor necrosis factor- $\alpha$ (TNF- $\alpha$ ) production in mouse macrophages [5]. The effective fiber to cell ratio found to elicit human macrophage cellular priming in this study was $1: 1$. Approximately a 1:2.5 fiber to cell ratio $\left(10^{6}\right.$ fibers $/ \mathrm{ml}$ ) was used as a low concentration in the rat macrophage studies reported by Blake et al., which may not have been high enough to prime the cellular response to zymosan above control following short fiber phagocytosis [7]. Secondly, luminol was used as the light enhancer in the present study, but lucigenin was used in the rat cell study. Luminol detects multiple reactive species compared to lucigenin, which is a superoxide specific light enhancer [16-18]. Most likely, superoxide is not the sole oxidant produced in glass fiber-exposed cells. Therefore, this experimental system may have an enhanced detection level compared to the past system. Finally, the result may reflect inherent differences in the oxidant production of rat and human alveolar macrophages. Human alveolar macrophages are reportedly more active than the rat in the production of reactive species following particulate exposure [19]. Whether this is the case with glass fibers would need further investigation.

It was found that no fiber length or concentration induced significant cellular membrane damage as assessed by cytosolic LDH release into the cellular supernatant although a concentration-dependent trend was apparent (Figure 1). Castranova et al. reported effects of metallic ions on particle-stimulated oxygen consumption and chemiluminescence in alveolar macrophages, i.e., functional assays, occurs at concentrations that do not affect the integrity of the cellular membrane [20]. Therefore, a reason for the lack of a significant LDH concentrationresponse may be that the cellular function is typically affected before membrane damage making the chemiluminescence response a more sensitive measure of cytotoxic effects.

In summary, this study reported no effects of fiber length over the range of $8-20 \mu \mathrm{m}$ on the human alveolar macrophage response. This conflicts with previous data where increased glass fiber concentration decreased chemiluminescence and increased LDH in rat alveolar macrophages but major length-dependent effects were evident. The reason for this discrepancy most likely is due to the larger diameter of human alveolar macrophages, resulting in the lack of frustrated phagocytosis in the present experimental system. Microscopic analysis verified these cells were able to completely engulf even the longest fiber sample tested $(20 \mu \mathrm{m})$ (Figure 3B). Blake et al. demonstrated cellular effects on rat alveolar macrophages (diameter $\sim 13 \mu \mathrm{m}$ ) using 17 and $33 \mu \mathrm{m}$ glass fibers, approximately $4-20 \mu \mathrm{m}$ longer than the cells (multiple rat macrophages were often observed adhered to a long fiber with the fiber appearing to protrude through the cellular membrane) [7]. In the present study, the longest fiber sample used was $20 \mu \mathrm{m}$, only $2 \mu \mathrm{m}$ longer than the human alveolar macrophage cellular diameter; therefore not a sufficient length to cause frustrated phagocytosis. Consequently, further studies are needed using fiber samples over a range of 20$40 \mu \mathrm{m}$ in length. Assuming similar mechanisms are involved in human and rat fiber phagocytosis, results most likely would agree with those reported by Blake and colleagues.

\section{Conclusion}

This study showed: 1.) an absence of length-associated cytotoxicity in primary human alveolar macrophages that was previously observed in rat alveolar macrophages treated in vitro with length-classified glass fibers 2.) a possible mechanism for this absence of length-dependent cytotoxicity may be the lack of frustrated phagocytosis in the human macrophages versus the rat 3.) human alveolar macrophages appear to be activated by glass fibers of monodisperse lengths at a fiber to cell ratio of approximately $1: 1$, while significant cytotoxicity was observed only at an excessively high fiber:cell ratio of 80:1. In conclusion, the use of monodisperse length-classified fiber samples will aid in the determination of specific fiber lengths that macrophages can engulf. In addition, these preliminary data may aid in the design of future in vivo experiments using fibrous particles.

\section{Methods \\ Fiber samples}

Bulk samples of JM-100 glass (Manville code 100 supplied by John Mansville Corporation) were first milled, aerosolized, and separated into length categories using dielectrophoresis as previously described $[3,4]$. The dielectrophoretic classifier was operated in a differential mode so that fibers with narrow length distributions were extracted in an air suspension at the end of the classifier. These length-classified fiber samples were collected on polycarbonate (Nuclepore) filters at rates up to $1 \mathrm{mg} /$ day. Fibers were scraped off the filters for microscopic analysis and for biological experiments. 

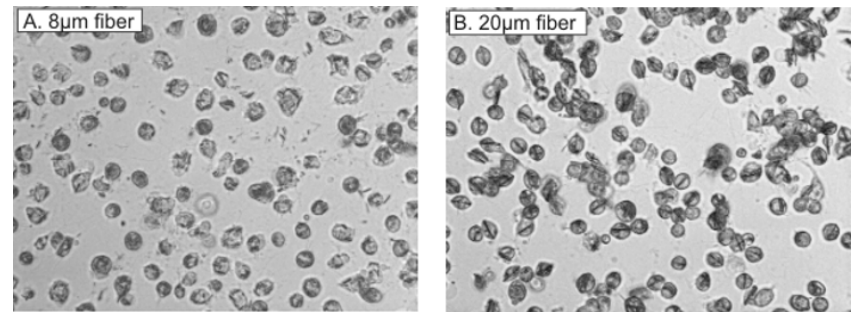

Figure 3

Microscopic analysis of primary human alveolar macrophages engulfing glass fibers. Panel A demonstrates the ability of the alveolar macrophages to engulf $8 \mu \mathrm{m}$ glass fibers. Panel B verifies effective phagocytosis of $20 \mu \mathrm{m}$ glass fibers by human alveolar macrophages.

Samples of the length-classified fibers were prepared for size and count analysis by adding weighed portions of the dusts to freshly filtered water. These samples were then diluted and filtered through polycarbonate filters. Measurements of length, width, and fiber count/mass were made using a JEOL JSM-6400 scanning electron microscope (Table 3). Measurements at each magnification were referenced to a National Institute of Standards and Technology electron microscopy standard rule.

All fiber samples were heat-sterilized and stored at $4-6^{\circ} \mathrm{C}$. Prior to each experiment, the fibers were suspended in sterile $\mathrm{Ca}^{+2}+\mathrm{Mg}^{+2}$ free phosphate-buffered saline (PBS).

\section{Human bronchoalveolar lavage}

Human alveolar macrophages were collected by a standard procedure at University of Pittsburgh. Briefly, a total of six male healthy subjects between the ages of 20 and 40 years were recruited for study. All signed a statement of informed consent approved by the University of Pittsburgh Institutional Review Board for Biomedical Research. Subjects were screened by history, physical examination, and spirometry. All subjects had to exhibit normal spirometry, and have no history of asthma or allergic diseases. Skin test responses to a panel of $15 \mathrm{com}$ mon allergens, plus histamine phosphate as a positive control (Greer Laboratories, Lenoir, NC), were determined using the prick-puncture method. Any subject demonstrating a positive skin test to an allergen was deemed to have atopic disease and was excluded. Bronchoscopy and bronchoalveolar lavage (BAL) were performed according to published methods [21-23]. Briefly, subjects were lightly sedated with midazolam $1.0 \mathrm{mg}$ and atropine $0.5 \mathrm{mg}$ IM, and meticulous local anesthesia of the nasopharynx was obtained using $4 \%$ cocaine solution, $1 \%$ lidocaine by gargle, and $1 \%$ benzocaine aerosol. Additional topical $1 \%$ lidocaine, via the bronchoscope work- ing channel, was applied to the vocal cords and airway mucosa. Total lidocaine dose delivered below the vocal cords was in all cases less than $200 \mathrm{mg}$. The bronchoscope was advanced to the right upper lobe, and wedged in a subsegment of the anterior segment. BAL was then performed using two aliquots of sterile saline solution $(0.9 \%$ $\mathrm{NaCl}, 37^{\circ} \mathrm{C}, 60 \mathrm{ml}$ each), recovered by hand suction. The bronchoscope was then moved to a subsegment of the right middle lobe, medial segment, and BAL was again performed. Cells were recovered from BAL fluid by centrifugation at $400 \times \mathrm{g}$ for 15 minutes and the resulting cell pellet was washed once with Hanks Balanced Salt Solution (Sigma, St Louis). Cells were counted using a hemocytometer and adjusted to $2 \times 10^{6}$ cells $/ \mathrm{ml}$ in Hanks Balanced Salt Solution. Differential cell counts were performed on Diff-Quick stained cytocentrifuge slides, and $\geq 300$ cells were identified as alveolar macrophages, lymphocytes, eosinophils, or neutrophils. Viability by trypan blue exclusion always exceeded $95 \%$, and cell populations were in excess of $95 \%$ alveolar macrophages, with the balance of the population comprised of lymphocytes. Neutrophils were rarely observed as were eosinophils. In healthy volunteers, purification over discontinuous Percoll gradients is unnecessary [23].

\section{Primary human alveolar macrophage zymosan-stimulated chemiluminescence}

Human alveolar macrophages were plated in a white opaque 96-well plate at $1 \times 10^{5} \mathrm{cells} /$ well in $200 \mu \mathrm{l}$ of sterile Eagle's modified essential medium (Biowhittaker, Walkersville, MD) without phenol red supplemented with $2 \%$ heat-inactivated fetal bovine serum, $1 \mathrm{mM}$ glutamine, 100 units $/ \mathrm{ml}$ penicillin/streptomycin, and 10 $\mathrm{mM}$ HEPES at $\mathrm{pH} 7.2$. After a 2 hour incubation at $37^{\circ} \mathrm{C}$, the adherent cells were washed three times with warm media. Glass fibers $(8,10,16$, or $20 \mu \mathrm{m})$ were then added to the cells at three different concentrations determined by fiber number: $4 \times 10^{5}, 4 \times 10^{6}$, and $4 \times 10^{7}$ fibers $/ \mathrm{ml}$. Cultures were brought to a final volume of $200 \mu \mathrm{l}$ and each treatment was done in duplicate. After 18 hours at $37^{\circ} \mathrm{C}$, the plate was centrifuged and the supernatant harvested and kept on ice for later analysis. Warm HEPESbuffered solution ( $\mathrm{pH}=7.4$ ) was then added to the alveolar macrophages on the plate and zymosan-stimulated chemiluminescence was measured. The lumigenic substance luminol ( $1 \mathrm{mM})$ was added to all wells of the assay plate followed by zymosan $(2 \mathrm{mg} / \mathrm{ml})$ or HEPES-buffered solution to a final volume of $220 \mu \mathrm{l} /$ well. Chemiluminescence was measured at $460 \mathrm{~nm}$ using a microplate chemiluminometer (ML 3000 Microtiter Plate Luminometer, Dynatech Laboratories, Chantilly, VA) for 30 minutes and total cpm per well per 30 minutes was recorded. Zymosan-stimulated chemiluminescence was calculated as the stimulated (with zymosan) value minus the corresponding unstimulated (without zymosan) value. 
Table 3: Physical Characteristics of JM-1 00 Glass Fibers Determined by SEM

\begin{tabular}{ccccc}
\hline Measurement & \multicolumn{4}{c}{ Length Classified Sample } \\
\cline { 2 - 5 } & $8 \mu \mathrm{m}$ & $10 \mu \mathrm{m}$ & $16 \mu \mathrm{m}$ & $20 \mu \mathrm{m}$ \\
\hline Lengtha $\left.^{2} \mu \mathrm{m}\right)$ & $8.38 \pm 2.89$ & $10.40 \pm 4.63$ & $16.22 \pm 1.90$ & $18.90 \pm 2.64$ \\
Width $(\mu \mathrm{m})$ & $0.69 \pm 0.29$ & $0.70 \pm 0.29$ & $1.08 \pm 0.43$ & $0.97 \pm 0.47$ \\
Fiber \#/mg & $11.4 \times 10^{7}$ & $8.95 \times 10^{7}$ & $2.2 \times 10^{7}$ & $2.92 \times 10^{7}$ \\
\hline
\end{tabular}

aLength measurements reported for the 16 and $20 \mu \mathrm{m}$ fiber samples exclude short fiber populations that occasionally adhered to the long fibers during classification. Prior to use, fiber suspensions were gently vortexed rather than sonicated to minimize release of these short fibers. Inclusion of short fibers in the analysis slightly lowered the mean fiber length in the distribution for the 16 and $20 \mu \mathrm{m}$ fiber samples. Note: Values for length and width are means \pm SD

\section{Lactate dehydrogenase activity}

$\mathrm{LDH}$ activity was determined in the culture supernatant by monitoring the $\mathrm{LDH}$ catalyzed oxidation of pyruvate coupled with the reduction of NAD at $340 \mathrm{~nm}$ using a commercial kit and a Cobas Mira Plus Transfer Analyzer (Roche Diagnostics Systems, Montclair, NJ).

\section{Microscopic analysis}

Photographs of human alveolar macrophages were taken using an Olympus IX70 inverted light microscope equipped with a Dage camera.

\section{Data analysis}

All data were analyzed using a one-way analysis of variance model (ANOVA) with a significant difference defined as $\mathrm{p} \leq 0.05$. Pairwise differences were assessed through appropriate contrasts.

\section{Abbreviations \\ BAL Bronchoalveolar Lavage}

JM-100 Manville Code 100 Glass Fibers

LDH Lactate Dehydrogenase

ND Not Detected

PBS Phosphate Buffered Saline

SEM Scanning Electron Microscope

SVFs Synthetic Vitreous Fibers

\section{Competing interests}

The author(s) declare that they have no competing interests.

\section{Authors' contributions}

PCZE carried out the in vitro cytotoxicity studies, analyzed the data, and drafted the manuscript. WJC, BTA, and MPC provided the human macrophages and provided assistance in study design and coordination. GJD and PB pro- vided the length-classified fiber samples and aided in fiber analysis. WJ performed the fiber analysis and provided assistance in the study design. TB conducted studies with the rat alveolar macrophages. VC conceived of the study, participated in its design and coordination and helped in manuscript preparation. All authors read and approved the final manuscript.

\section{Disclaimer}

The findings and conclusions in this report are those of the authors and do not necessarily represent the views of the National Institute for Occupational Safety and Health.

\section{References}

I. Hesterberg TW, Hart GA: Synthetic vitreous fibers: a review of toxicology research and its impact on hazard classification. Crit Rev Toxicol 200I, 3 I(I): I-53.

2. Hesterberg TW, Hart GA, Chevalier J, Miller WC, Hamilton RD, Bauer J, Thevenaz P: The importance of fiber biopersistence and lung dose in determining the chronic inhalation effects of X607, RCFI, and chrysotile asbestos in rats. Toxicol Appl Pharmacol 1998, I 53:68-82.

3. Baron PA, Deye GJ, Fernback J: Length separation of fibers. Aerosol Sci Technol 1994, 21:I79-192.

4. Deye GJ, Gao P, Baron PA, Fernback J: Performance evaluation of a fiber length classifier. Aerosol Sci Technol 1999, 30:420-437.

5. Ye J, Shi X, Jones W, Rojanasakul Y, Cheng N, Schwegler-Berry D, Baron P, Deye GJ, Li C, Castranova V: Critical role of glass fiber length in TNF-alpha production and transcription factor activation in macrophages. Am J Physiol I999, 276:L426-434.

6. Ye J, Zeidler P, Young S-H, Martinez A, Robinson VA, Jones W, Baron $P$, Shi $X$, Castranova V: Activation of mitogen-activated protein kinase p38 and extracellular signal-regulated kinase is involved in glass fiber-induced tumor necrosis factor- $\alpha$ production in macrophages. J Biol Chem 200I, 276:5360-5367.

7. Blake T, Castranova V, Schwegler-Berry D, Baron P, Deye GJ, Li C, Jones W: Effect of fiber length on glass microfiber cytotoxicity. J Toxicol Environ Health A 1998, 54:243-259.

8. Archer VE: Carcinogenicity of fibers and films: a theory. Med Hypotheses 1979, 5:1257-1262.

9. Dorger M, Munzing S, Allmeling A-M, Messmer K, Krombach F: Differential responses of rat alveolar and peritoneal macrophages to man-made vitreous fibers in vitro. Environ Res 2001 , 85:207-214.

10. Oberdorster G: Toxicokinetics and effects of fibrous and nonfibrous particles. Inhal Toxicol 2002, I 4:29-56.

II. Vallyathan V, Mega JF, Shi X, Dalal NS: Enhanced generation of free radicals from phagocytes induced by mineral dusts. Am J Respir Cell Mol Biol 1992, 6:404-4I3.

12. Hart GA, Kathman LM, Hesterberg TW: In vitro cytotoxicity of asbestos and man-made vitreous fibers: roles of fiber length, diameter and composition. Carcinogenesis 1994, I 5:971-977. 
13. Hesterberg TW, Barrett JC: Dependence of asbestos- and mineral dust-induced transformation of mammalian cells in culture on fiber dimension. Cancer Res 1984, 44:2170-2180.

14. Miller BG, Searl A, Davis JMG, Donaldson K, Cullen RT, Bolton RE, Duncan B, Soutar CA: Influence of fibre length, dissolution, and biopersistence on the production of mesothelioma in the rat peritoneal cavity. Am Occup Hyg 1999, 43:155-166.

15. Lapp NL, Lewis D, Schwegler-Berry D, Abrons H, Castranova V: Bronchoalveolar lavage in asymptomatic underground coal miners. Proceedings of the 3rd Symposium on Respirable Dust in the Mineral Industries Society for Mining, Metallurgy and Exploration: 1991; Colorado 1991:159-169.

16. DeChatelet LR, Long GD, Shirley PS, Bass DA, Thomas MJ, Henderson FW, Cohen MS: Mechanism of the luminol-dependent chemiluminescence of human neutrophils. J Immunol 1982, I29:1589-1593.

17. Gyllenhammar $\mathrm{H}$ : Lucigenin chemiluminescence in the assessment of neutrophil superoxide production. I Immunol Meth 1987, 97:209-2।3.

18. Ischiropoulos $\mathrm{H}$, Kumae T, Kikkawa Y: Effect of interferon inducers on superoxide anion generation from rat liver microsomes detected by lucigenin chemiluminescence. Biochem Biophys Res Commun 1989, 161:1042-1048.

19. Rahman Q, Norwood J, Hatch G: Evidence that exposure of particulate air pollutants to human and rat alveolar macrophages leads to differential oxidative response. Biochem Biophys Res Commun 1997, 240:669-672.

20. Castranova V, Bowman L, Reasor MJ, Miles PR: Effects of heavy metal ions on selected oxidative metabolic processes in rat alveolar macrophages. Toxicol Appl Pharmacol 1980, 53: I4-23.

21. Calhoun W], Dick EC, Schwartz LB, Busse WW: A common cold virus, rhinovirus 16, potentiates airway inflammation after segmental antigen bronchoprovocation in allergic subjects. $J$ Clin Invest 1994, 94:2200-2008.

22. Calhoun WJ, Jarjour NN: Macrophages and macrophage diversity. In Asthma and Rhinitis Edited by: Busse W, Holgate S. Cambridge: Blackwell Science; 1995.

23. Calhoun WJ, Reed HE, Moest DR, Stevens CA: Enhanced superoxide production by alveolar macrophages and airspace cells, airway inflammation, and alveolar macrophage density changes follow segmental antigen bronchoprovocation in allergic subjects. Am Rev Respir Dis 1992, 145:317-325.
Publish with Biomed Central and every scientist can read your work free of charge

"BioMed Central will be the most significant development for disseminating the results of biomedical research in our lifetime. "

Sir Paul Nurse, Cancer Research UK

Your research papers will be:

- available free of charge to the entire biomedical community

- peer reviewed and published immediately upon acceptance

- cited in PubMed and archived on PubMed Central

- yours - you keep the copyright 